\title{
Efficiently Passive Monitoring Flow Bandwidth ${ }^{\star}$
}

\author{
Zhiping Cai, Jianping Yin, Fang Liu, Xianghui Liu, and Shaohe Lv \\ School of Computer, National University of Defense Technology, \\ Changsha, 410073, China \\ caizhiping_nudt@163.com, jpyin@nudt.edu.cn, \\ fangl_nudt@163.com, liuxh@tom.com, chi.shaohe@gmail.com
}

\begin{abstract}
Using the flow-conservation law, we could reduce the number of activated monitor agents used to monitor link bandwidth usage. In this paper, we address the problem of efficiently passive monitoring flow bandwidth based on flow-conservation, which could be reduced to weak vertex cover problem. And the weak vertex cover problem is NP-hard. We give an approximation algorithm with approximation ratio 2 to solve the problem. The effectiveness of our monitoring algorithm is validated by simulations evaluation over a wide range of network topologies.
\end{abstract}

\section{Introduction}

Bandwidth utilizations are critical for numerous important network management tasks. Some novel tools and infrastructures for measuring network bandwidth have been developed and proposed by researchers and industries.

The number of placed monitors of a monitoring system should be kept as small as possible in order to reduce the deployment cost and the actual monitoring operating cost [1]. Several measurements over backbone routers show each IP router satisfies a flow-conservation law that, the sum of the traffic flowing into router is approximately the same as those of the traffic flowing out [2]. The problem of efficiently monitoring the network flowing based on flow-conservation could be reduced to the weak vertex cover problem.

The paper is structured as follows. The weak vertex cover problem is brought forward, and some approximation results for the weak vertex cover problem are listed in the section 2. In next section, we give an approximation algorithm to solve the weak vertex cover problem with approximation ratio 2 . The effectiveness of our monitoring algorithm is validated by simulations evaluation over a wide range of network topologies in section 4 . And we depict our further research in the last section.

\section{Weak Vertex Cover Problem}

The problem of efficiently passive monitoring the network flowing based on flowconservation could be reduced to the weak vertex cover problem, which is NPhard $[2],[3]$.

\footnotetext{
^ This work is supported by the National Natural Science Foundation of China under Grant No. 60373023.
} 
Definition 1. (Weak Vertex Cover) Given an undirected graph $G=(V, E)$, where $\forall v \in V, d(v) \geq 2$ holds, we say $S \subseteq V$ is a weak vertex cover Set of $G$, if and only if every edge in $G$ can be marked by performing the following three steps:

(1) Mark all edges that are incident on vertices in $S$;

(2) Mark the edge if it is the only unmarked edge among all of the edges that are incident on the same vertex;

(3) Repeat step (2) until no new edge can be marked.

For solving the problem of finding the minimum weak vertex cover set, Xianghui Liu et al. [3] brought forward a greedy approximation algorithm which gives an approximation ratio $2(1+\ln d)$, where $d=\max _{v \in V}\{d(v)\}$. And Xianghui Liu et al. [4] proved that the weak vertex cover problem is NP-complete. Yong Zhang et al. [5] gave an approximation algorithm with approximation ratio $1+\ln d$. Zhiping Cai et al. [6] gave an approximation preserving reduction from the vertex cover problem to the weak vertex cover problem. Due to this reduction, it implied that it is difficult to get an approximation algorithm with approximation ratio small than 2 .

\section{An Approximation Algorithm}

We give a 2-approximation algorithm for the weak vertex cover by using the primal-dual method for approximation algorithms, which has been used to derive approximation algorithms for network design problems [7-9].

At the beginning, we give some inequalities that will be needed in proving the performance guarantees of the algorithms and in giving the integer programming formulation. In fact, we could get different primal-dual algorithms by using different integer programming formulation [9].

Given a subset $S$ of vertices, let $E[S]$ denote the subset of edges that have both endpoints in $S$. Let $G[S]$ denote the subgraph $(S, E[S])$ induced by $G$, and let $d_{s}(v)$ denote the degree of $v$ in $G[S]$. We let $b(S)=|E[S]|-|S|+1$ and $b(V)=|E|-|V|+1$. Observe that if $F$ is a weak vertex cover for $G$, then $F \cap S$ is clearly a weak vertex cover for $G[S]$. Hence we have following theorem. The details of this proof are omitted due to space limitations.

Theorem 1. Let $F$ be any weak vertex set. Then for any $S \subseteq V, E[S] \neq 0$,

$$
\sum_{v \in F \cap S}\left(d_{s}(v)-1\right) \geq|E[S]|-|S|+1=b(S)
$$

By Theorem 1, the integer programming formulation of the weak vertex cover problem is the following:

$$
\operatorname{Min} \sum_{v \in V} w_{v} x_{v}
$$


Subject to:

$$
\begin{array}{cc}
\sum_{v \in S}\left(d_{s}(v)-1\right) x_{v} \geq b(S) & S \subseteq V, E[S] \neq 0 \\
x_{v} \in\{0,1\} & v \in V .
\end{array}
$$

We construct a feasible solution to the dual of the linear programming relaxation of (IP). The linear programming relaxation is

$$
\operatorname{Min} \sum_{v \in V} w_{v} x_{v}
$$

Subject to:

$$
\begin{array}{cc}
\sum_{v \in S}\left(d_{s}(v)-1\right) x_{v} \geq b(S) & S \subseteq V, E[S] \neq 0 \\
x_{v} \geq 0 & v \in V .
\end{array}
$$

And its dual is

$$
\operatorname{Max} \sum_{S} b(S) y_{s}
$$

Subject to:

$$
\begin{array}{cc}
\sum_{v \in S}\left(d_{s}(v)-1\right) y_{s} \leq w_{v} & v \in V \\
y_{s} \geq 0 & S \subseteq V, E[S] \neq 0 .
\end{array}
$$

Then an approximation algorithm is given as follows:

Algorithm WeakCover $(G=(V, E))$ :

1. $y=0 ; F=0 ; l=0$;

2. $V^{\prime}=V ; E^{\prime}=E$;

3. While $F$ is not a WVC for $G$
(a) $l=l+1$;
(b) Recursively remove degree one vertices and incident edges from $V^{\prime}$ and $E^{\prime}$
(c) $S=\operatorname{Endblock}\left(V^{\prime}, E^{\prime}\right)$;
(d) Increase $y_{S}$ until $\exists v_{l} \in S$, s.t. $\sum_{T: v_{l} \in T}\left(d_{T}\left(v_{l}\right)-1\right) y_{T}=w_{v_{l}}$
(e) $F=F \cup\left\{v_{l}\right\}$
(f) Remove $v_{l}$ from $V^{\prime}$ and attached edges from $E^{\prime}$.

4. For $(j=l ; j>0 ; j--)$

(a) if $F-\left\{v_{j}\right\}$ is a WVC then $F=F-\left\{v_{j}\right\}$

5. $F^{\prime}=F$

And the Endblock procedure is defined as follows:

Procedure Endblock $\left(V^{\prime}, E^{\prime}\right)$ :

1. Return the vertices of an endblock of $\left(V^{\prime}, E^{\prime}\right)$;

Note that the worst-case time complexity of the algorithm can be shown to be $O(|V||E|)$.And the algorithm computes a 2 approximation for the Weak Vertex Cover problem.The details of this proof are omitted due to space limitations. 


\section{Simulations}

The effectiveness of our monitoring algorithm is validated by simulations evaluation over a wide range of network topologies generated using the Waxman Model [10]. Simulation result has been omitted due to paper size limitations and the simulation shows the our proposed algorithmic solutions are not only theoretically sound but also they could give significant benefits over naive solutions in practice for a wide variety of realistic network topologies.

\section{Conclusions}

In this paper, we have addressed the problem of efficiently passive monitoring flow bandwidth. This problem could be abstracted to the weak vertex cover problem, which is NP-hard. We have proposed a 2-approximation algorithm to solve Weak Vertex Cover problem. Finally, we have verified the effectiveness of our approximation algorithms through simulations evaluation.

Further research would be conducted to exploit knowledge of traffic flows in the network to further reduce the required overhead for monitoring flow bandwidth.

\section{References}

1. Kyoungwon Suh, Yang Guo, Jim Kurose, and Don Towsley. Locating Network Monitors: Complexity, Heuristics, and Coverage. In Proc. IEEE INFOCOM 2005.

2. Breitbart Y., Chan CY., Garofalakis M., Rastogi R., Siberschatz A.: Efficiently Monitoring Bandwidth and Latency in IP Networks. In Proc. IEEE INFOCOM 2001.

3. Xianghui Liu, Jianping Yin, Lele Tang: Analysis of Efficient Monitoring Method for the Network Flow. Journal of Software, 2003,14(2): 300-304(in Chinese with English abstract).

4. Xianghui Liu, Jianping Yin, Xicheng Lu: A Monitoring Model for Link Bandwidth Usage of Network Based on Weak Vertex Cover. Journal of Software, 2004,15(4): 545-549(in Chinese with English abstract).

5. Yong Zhang and Hong Zhu: Approximation Algorithm for Weighted Weak Vertex Cover. Journal of Computer Science and Technology, 2004,19(6): 782-786.

6. Zhiping Cai, Jianping Yin, Fang Liu, Xianghui Liu, Shaohe Lv: Efficiently Monitoring Link Bandwidth in IP Networks. In Proc. IEEE GLOBECOM 2005.

7. Dorit S. Hochbaum: Approximation Algorithm for NP-Hard Problems. PWS Publishing Company,1997.

8. A. Becker and D. Geiger: Approximation Algorithms for the Loop Cutest Problem. In Proc. $10^{\text {th }}$ Conference on Uncertainty in Artificial Intelligence.

9. F. A. Chudak, M. X. Goemans, D. S. Hochbaumn, and D. P. Williamson: A PrimalDual Interpretation of Two 2-Approximation Algorithms for the Feedback Vertex Set Problem in Undirected Graphs. Operations Research Letters, 1998, 22 :111-118.

10. B.M.Waxman: Routing of Multipoint Connections. IEEE Journal on Selected Areas in Communications, 1988, 6(9):1617-1622. 